\title{
LEVANTAMENTO DE PESQUISAS REALIZADAS NA APA DE MARICÁ - RJ
}

\author{
Fernanda Kreischer Bandeira Diniz ${ }^{1}$ \\ Angela Loureiro Gonçalves da Silva ${ }^{1}$ \\ Bruno da Costa Dias ${ }^{1}$ \\ Luciana Silva de Assis ${ }^{1}$ \\ Mariana Samor Lopes ${ }^{1}$ \\ Monique Cerqueira de Souza ${ }^{2}$ \\ Rebeca Oliveira Castro ${ }^{1}$ \\ Douglas de Souza Pimentel $^{3}$
}

\section{RESUMO}

A Área de Proteção Ambiental (APA) de Maricá, criada em 1984 e com 496 hectares de vegetação de restinga, é uma Unidade de Conservação (UC) ameaçada por conta de várias intervenções, principalmente projetos de construção civil. Seu plano de manejo foi aprovado por decreto em 2007. Existe a necessidade de se coletar dados e aumentar o nível de conhecimento da área, assim como sobre as populações que nela residem, viabilizando assim a preservação ambiental. Por isso, o presente estudo visa agregar informações sobre a produção de pesquisas relacionadas à fauna, flora, geografia e informações socioambientais. Foi realizado um levantamento bibliográfico sobre a APA de Maricá envolvendo trabalhos desde a década de 1920 e, em seguida, a montagem de um banco de dados onde as referencias foram classificadas de acordo com o tipo, ano de publicação e área de conhecimento. A partir do levantamento foi possível identificar que a maior parte da pesquisa realizada na área foi publicada em formato de livro ou artigo em revista científica. Os temas mais abordados eram voltados para fauna e flora, onde os grupos mais averiguados foram os artrópodes. A maioria das pesquisas ocorreu nos anos de 1995, 2010 e 2005. Após o decreto de criação da APA o número de pesquisas aumentou e, em 2007, devido à suspensão da construção de um resort na APA de Maricá, elevou-se a quantidade de pesquisas direcionadas para a área socioambiental. Embora já existam trabalhos publicados desde 1924, há campos de conhecimento, como limnologia e educação ambiental, deficientes de estudos. Além disso, a APA contém um plano de manejo escasso de informações, logo, há a necessidade de se reformular esse plano, a partir dos novos dados e pesquisas sobre a UC.

Palavras Chave: Área de proteção Ambiental, Maricá - RJ, Unidades de Conservação.

\section{ABSTRACT}

The Environmental Protection Area of Maricá, established in 1984, with 496 acres of salt marsh vegetation, which is currently an endangered protected area extinction due to the advancement of construction, had its management plan enacted in 2007. There is a need to collect data and increase knowledge of the area level and the populations living in it, thus enabling environmental preservation. So the present study, aims to add information on fauna,

\footnotetext{
${ }^{1}$ Discente de Pós Graduação em Biologia Marinha e Ambientes Costeiros (UFF)

${ }^{2}$ Discente de Especialização em Engenharia Ambiental, Faculdade Maria Thereza - FAMATH

${ }^{3}$ Docente da Pós Graduação em Biologia Marinha e Ambientes Costeiros (UFF)

E-mail para contato: fernandakbd@gmail.com
} 
flora, local geography and environmental studies. A literature review on the Environmental Protection Area of Maricá has been made since the 1920s, then the elaboration of a database, where the references were classified according to the type, year of publication and area of knowledge. From the survey we observed that most of the research conducted in the conservation unit was published in the format of book or article in journal, the most discussed topics were focused on fauna-flora, where the most investigated groups were arthropods. The more research was in 1995, 2010 and 2005. After the decree of creation of the APA the number of searches increased, and in 2007, due to the suspension of the construction of a resort in the protected area of Maricá, increased the amount of research directed to socioenvironmental area. Although there are works published since 1924, there are areas, such as limnology and environmental education, disabled of studies. In addition, the protected area contains a management plan scarce of information, so there is the need to reformulate the plan, based on new data and research on the conservation area.

Keywords: Marica- RJ, Environmental Protection Area, Protected Areas

\section{INTRODUÇÃO}

A intensificação da expansão urbana para a Região dos Lagos do Estado do Rio de Janeiro ocorreu a partir da década de 1970 quando a indústria imobiliária trouxe radicais mudanças para os ecossistemas lagunares e fluviais e seu entorno, com o lançamento indiscriminado de esgotos domésticos e com o desmatamento de áreas de restingas, dunas e florestas (AZEVEDO \& SERRANO, 2008).

A atividade urbana desestabiliza progressivamente os componentes biológicos e da paisagem, com consequente degradação oriunda dos impactos da expansão urbana. Andrade (1967) demonstrou que há uma correlação significativa entre o aumento do tamanho das ilhas de vegetação e a maior variabilidade de ambientes que aumenta a quantidade de recursos no seu interior, diminuindo a probabilidade de extinção de espécies.

A Área de Proteção Ambiental (APA) de Maricá, criada em 1984, é a mais antiga APA sob administração estadual, sendo inicialmente gerenciada pela Fundação Estadual de Engenharia do Meio Ambiente (FEEMA), atualmente Instituto Estadual do Ambiente (INEA). A área protegida abrange 496 hectares de vegetação de restinga em uma estreita faixa que se estende no sentido leste-oeste, entre o Oceano Atlântico e a Laguna de Maricá, no sentido sul-norte (SEMAM/IEF, 1994). A área em questão é constituída por extenso cordão litorâneo, com cerca de $20 \mathrm{~km}$ de extensão, caracterizado geologicamente e, em termos de fisionomia vegetal, como formação de restinga (HOLZER et al., 2004).

Recentemente a faixa de restinga da região de Maricá passou por um processo de degradação intensa devido à exploração de areia e turfa, circulação de veículos, retirada de espécies vegetais, deposição de lixo e ainda pelo parcelamento e edificações (CRICHYNO et al. 2003).

Segundo Loureiro et al. (2010), a APA de Maricá está localizada no litoral do Estado do Rio de Janeiro numa faixa arenosa com cerca de $8 \mathrm{~km}$ de extensão, desde a Ponta Negra até a Ponta de Itaipuaçú. Existem formações de restinga (Fazenda São Bento da Lagoa) e 
tabuleiros costeiros cobertos por vegetação de mata atlântica em falésias esculpidas pelo mar em terrenos terciários (Ilha Cardosa, Ponta do Fundão e o Morro do Mololô). Nos cordões arenosos a vegetação apresenta altura variável entre $2 \mathrm{~m}$ e $5 \mathrm{~m}$, com trechos de vegetação típica de restinga como cactos, bromélias, espécies diversas de gramíneas e arbustos de baixo porte. Este ecossistema abriga flora e fauna significativa, incluindo aves migratórias e espécies endêmicas e ameaçadas de extinção, além de possuir sítios arqueológicos a serem estudados, consistindo em um rico patrimônio ambiental, cultural, arqueológico e científico (MATIAS et al. 2007).

A APA de Maricá, com plano de manejo decretado em 2007, abrange uma área de proteção ambiental ameaçada pela ocupação humana devido ao avanço dos projetos de construção civil, além de diversas outras interferências que vem alterando significativamente sua composição florística e vegetacional, tais como: retirada de areia das dunas, coleta de mudas para comercialização, queimadas, cortes sistemáticos de espécimes para obtenção de lenha, dentre outros fatores (RIZINI et al. 1990). A situação ambiental é agravada ainda pela presença da comunidade pesqueira de Zacarias, atuante na localidade desde o século XVIII. Um possível e recente projeto de implantação de um resort, poderá descaracterizar ainda mais a APA, a única com uma grande área de vegetação de restinga preservada do município (ARAÚJO et al. 2008).

A importância dos estudos descritivos para a preservação ambiental e manejo de UCs reside no fornecimento de dados sobre a dinâmica de comunidades, facilitando a escolha de espécies cujas populações mereçam estudos mais detalhados, objetivando a preservação de espécies raras e o aumento de conhecimento sobre estratégias bem sucedidas (PEREIRA et al. 2001).

Tendo em vista essa problemática, este artigo visa quantificar trabalhos de diversos domínios, colaborando para a detecção das áreas com maior deficiência de conhecimento na área de estudo.

\section{MATERIAL E MÉTODOS}

O levantamento bibliográfico foi realizado no site de busca Google Acadêmico e na plataforma de trabalhos científicos da Capes utilizando as seguintes palavras-chaves: APA (de) Maricá, Área de proteção ambiental (de) Maricá. Também foi usado o relatório presente na base do Instituto Estadual do Ambiente (INEA) dos trabalhos realizados na unidade. A pesquisa permitiu a construção de um banco de dados com as referências sobre as pesquisas realizadas na APA de Maricá desde a década de 1920, quando surgiram as primeiras publicações científicas relacionadas à área de estudo. Após o levantamento, as referências foram categorizadas de acordo com o tipo (publicações em revistas científicas ou livros, publicações em eventos científicos, teses, dissertações, monografias de graduação e pósgraduação); o ano de publicação e área de conhecimento (flora, fauna, limnologia, geologia, geografia, socioambiental, antropologia e educação ambiental). 


\section{RESULTADOS}

\section{a) Tipologia de publicações}

Toda pesquisa envolve atividades diversas de comunicação e produz pelo menos uma publicação formal. Tais publicações variam no formato: relatórios, trabalhos apresentados em congressos, palestras, artigos de periódicos, livros e outros (CAMPELLO, 2000).

Como é possível observar na Figura 1, a maior parte das pesquisas realizadas na área que inclui a atual APA de Maricá, foi publicada no formato de livro ou artigo em revista científica (50\%), seguido de publicações em eventos científicos (32\%), dissertações de mestrado $(7 \%)$, teses de doutorado $(6 \%)$, trabalhos de conclusão de curso de pós graduação $(3 \%)$ e monografias $(2 \%)$.

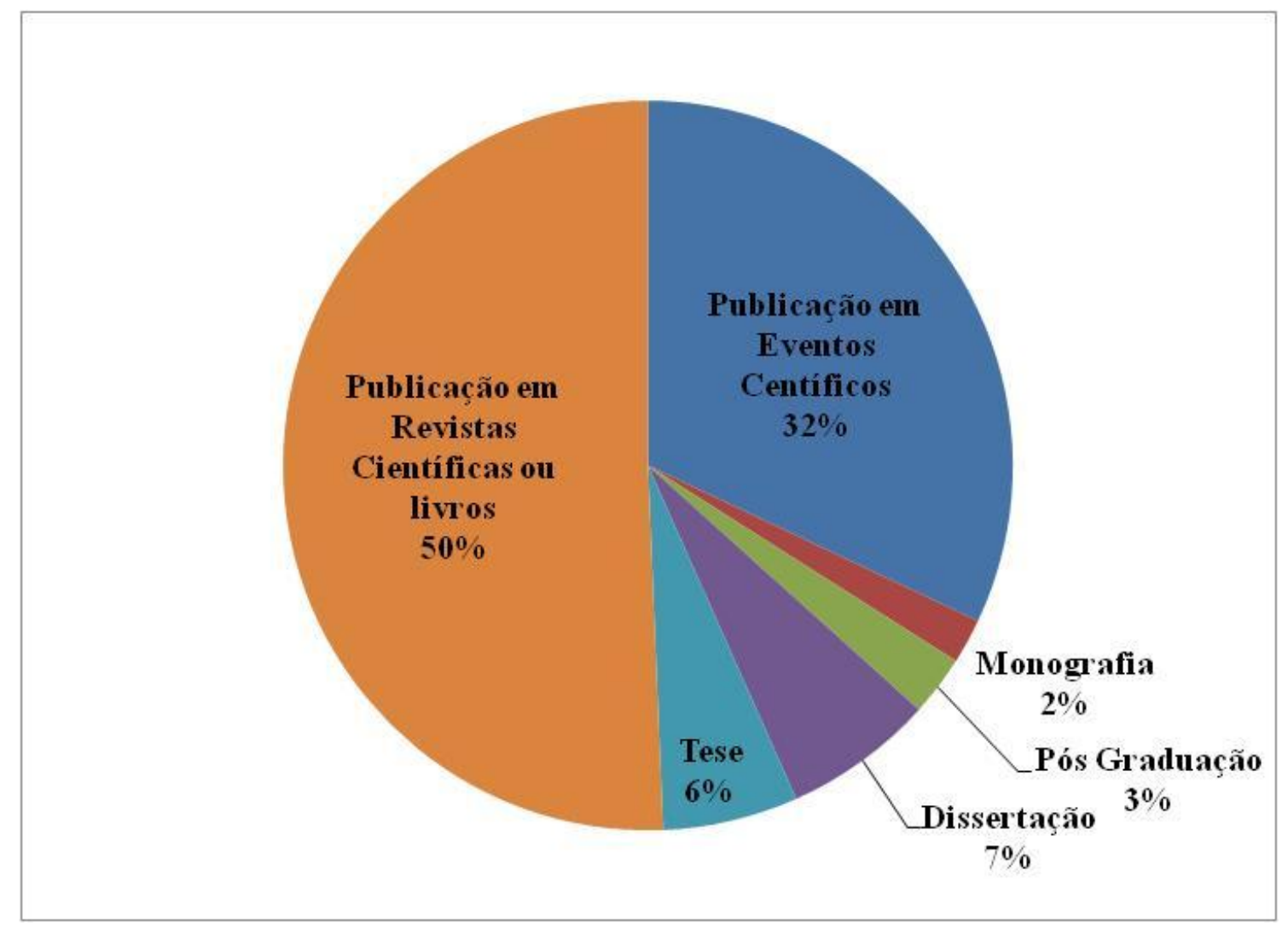

Figura 1 - Distribuição das pesquisas realizadas na área da atual APA de Maricá por tipologia

\section{b) Eixos temáticos das pesquisas}

A Figura 2 mostra que a maior parte das pesquisas ocorreram no eixo temático faunaflora (37.60\% de estudos de fauna, $37.60 \%$ de estudos de flora e $0.41 \%$ de ambos os eixos), seguido de $12.81 \%$ de estudos socioambientais e antropológicos, $9.92 \%$ geografia e geologia 
( $0.41 \%$ envolvendo os eixos sociambiental e geologia), $0.83 \%$ em educação ambiental e $0.41 \%$ limnologia.

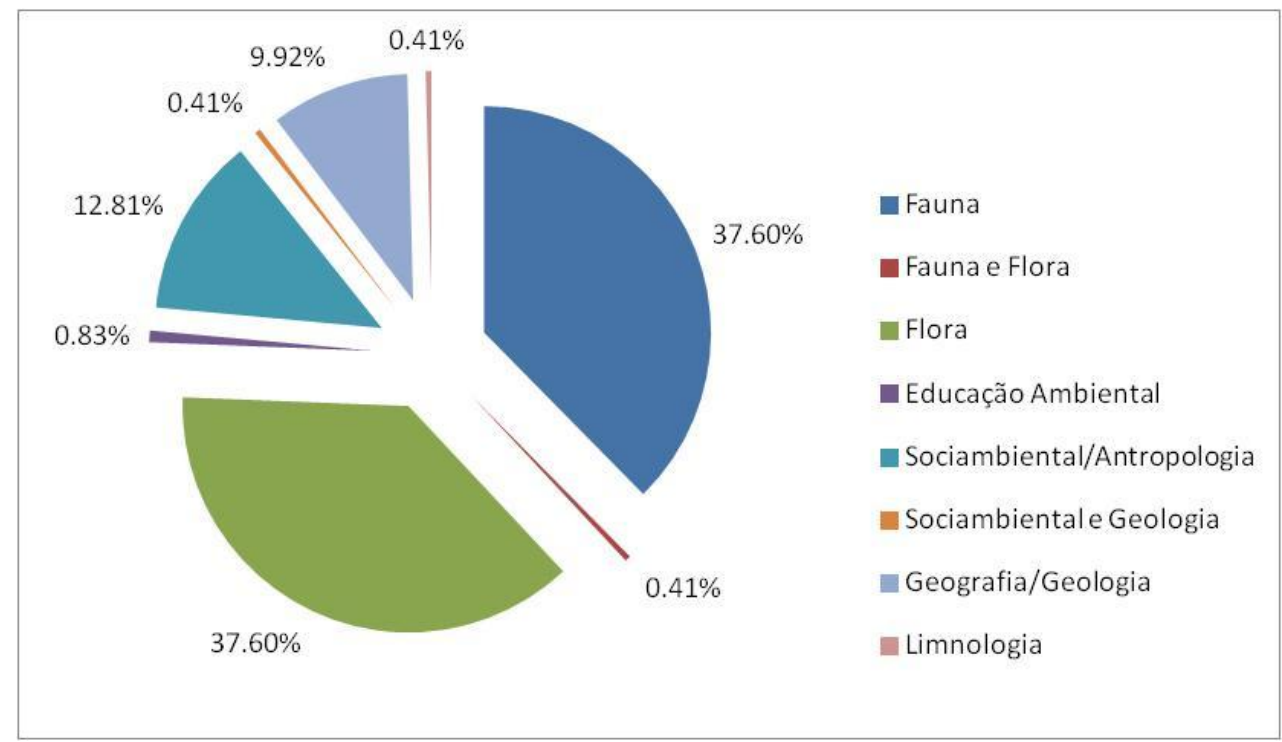

Figura 2 - Distribuição das pesquisas realizadas na na área da atual APA de Maricá por eixo temático

Os estudos de educação ambeintal tiveram seu início tardiamente na UC e ainda tem pouca representatividade. O primeiro foi feito somente onze anos após a criação da UC, em 2005 e o segundo, em 2012. O mesmo ocorre com a limnologia que possui somente uma publicação.

A fim de se entender quais os filos e classes mais estudados na APA de Maricá, todos os trabalhos foram divididos em categorias (Figura 3). Os artrópodes foram o grupo mais estudado (43\%), seguido de répteis (17\%) e mamíferos $(14 \%)$. 


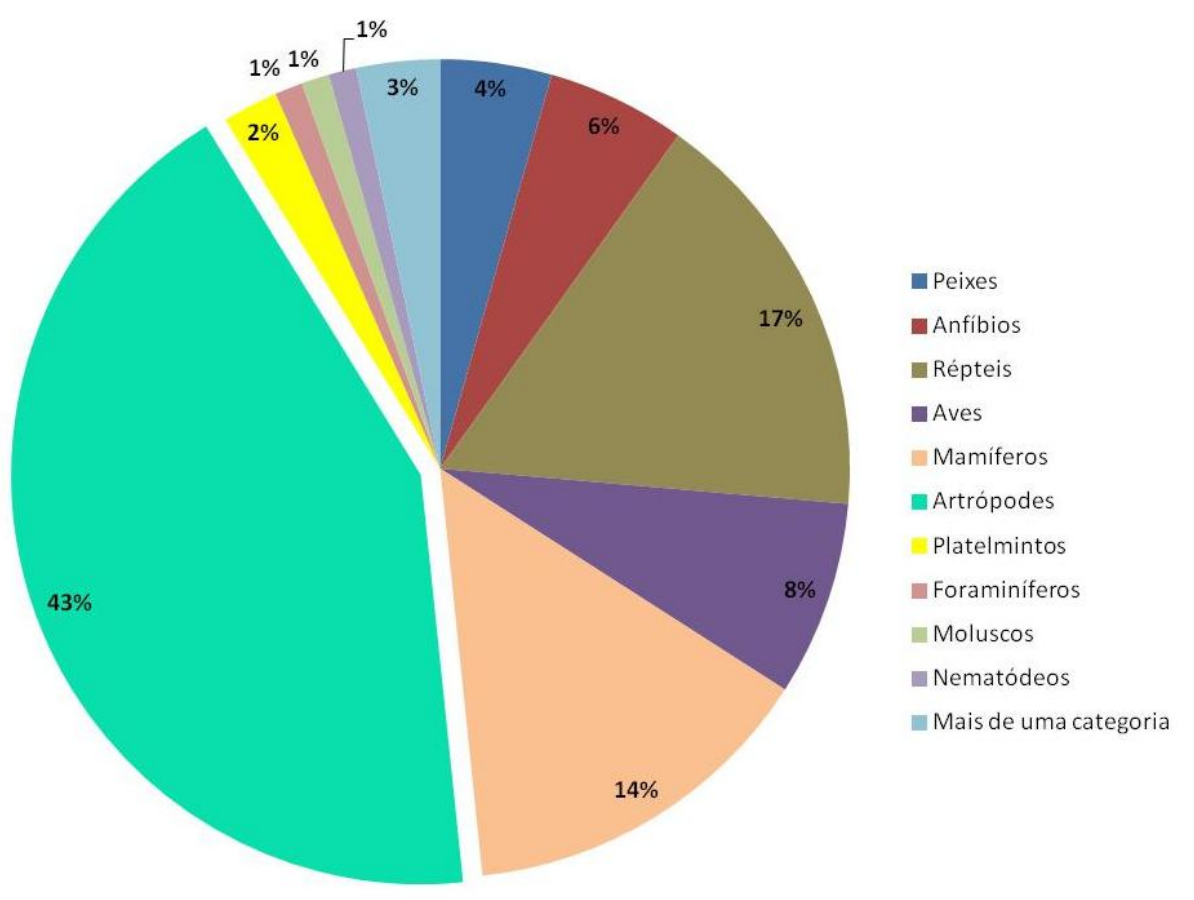

Figura 3 - Distribuição das pesquisas realizadas na fauna por filos e classes

Todos os trabalhos feitos com répteis foram de lagartos e quase todos do mesmo grupo de pesquisa da Universidade Estadual do Rio de Janeiro - UERJ (ROCHA, 1985; RIBAS, 1995; VRCIBRADIC, 1992 e WINCK, 2012).

\section{c) Números de pesquisas por ano}

Como é possível observar na Figura 4, os estudos começaram antes mesmo da criação da APA, em 1984. Os anos com maiores número de pesquisa foram 1995 (17), seguido de 2010 (15) e 2005 (14).

Observa-se que após o decreto de criação da APA o número de pesquisas aumentou muito. 


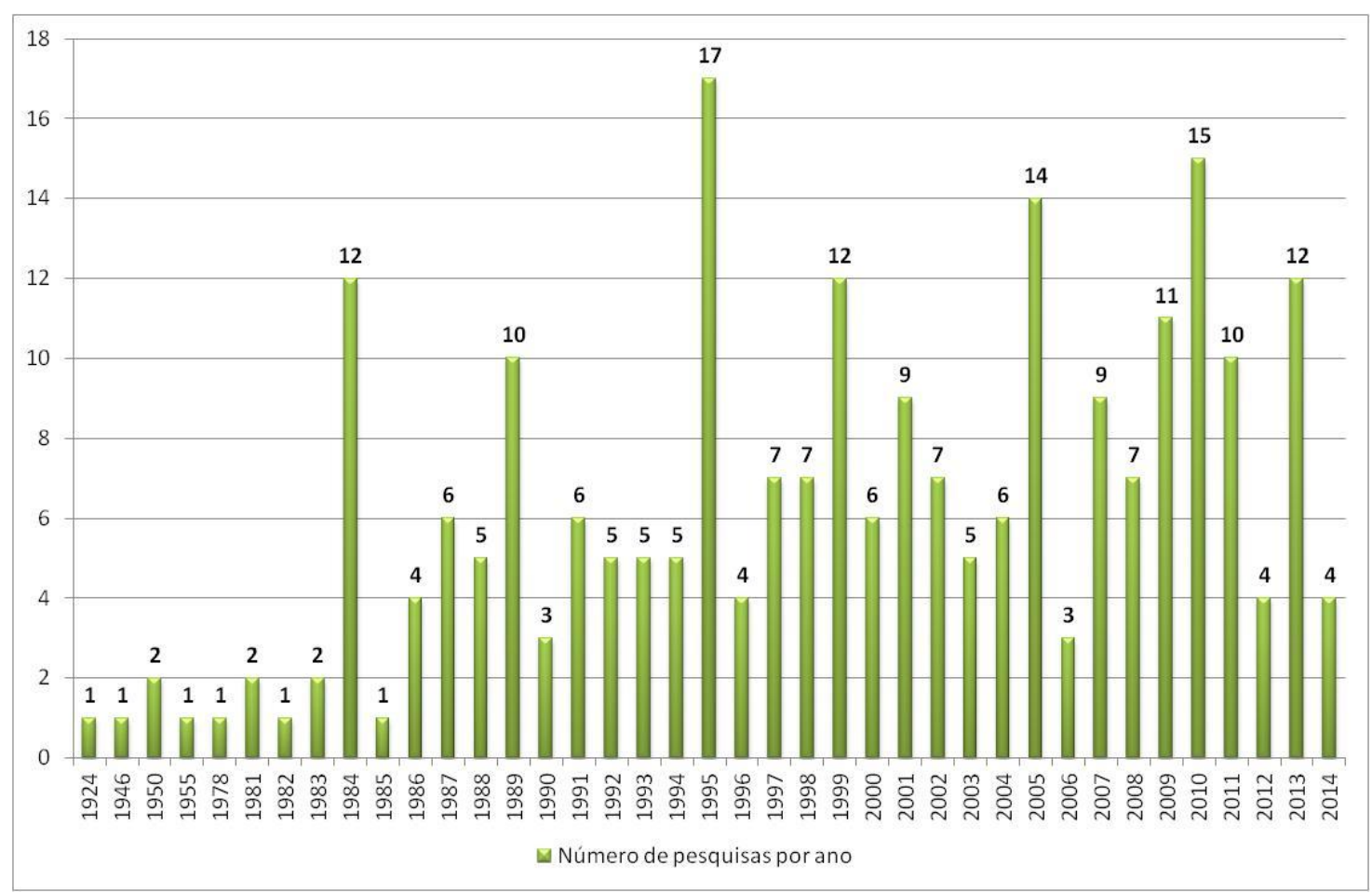

Figura 4 - Distribuição por anos das pesquisas realizadas na APA de Maricá

Em maio de 2007 um dos administradores da empresa portuguesa Dico Harisa Obrum, Miguel de Almeida, lançou o empreendimento do mega resort na APA de Maricá durante o Salão Imobiliário de Madri (FIOCRUZ e FASE, 2015).

Pelos projetos do consórcio, seria construído um resort (constituído de dois condomínios residenciais, espaço comercial e de alimentação, um campo de golfe para provas de nível internacional, um centro equestre, uma marina para 1.100 barcos e um centro de tênis) para 95 mil habitantes, em um lote de 841 hectares com $8 \mathrm{~km}$ de praia e 5 de lagoa, gerando um custo de oito bilhões de reais (FIOCRUZ e FASE, 2015).

O grupo de construtoras só iniciou o pedido de licenciamento ambiental do empreendimento junto ao INEA em Maio de 2011 (ALENCAR, 2011), porém em Dezembro de 2013 uma Liminar da Justiça Estadual suspendeu o licenciamento ambiental do empreendimento (DA MATTA, 2013).

Esse cenário fez com que, desde 2007, fossem publicadas pesquisas do eixo temático socioambiental mostrando a preocupação da comunidade científica em preservar a comunidade tradicional de pescadores ali presente e o meio ambiente (UEHARA-PRADO \& FONSECA, 2007; LOUREIRO et al., 2010; DOMINGUES, 2010; MATIAS \& CORREA, 2010; MATIAS et al., 2010; COYUNJI, 2013; CRICHYNO et al, 2013). 


\section{d) A questão do plano de manejo}

A APA de Maricá possui um plano de manejo instituido pelo decreto no 41.048 de 04 de dezembro de 2007. Esse documento é extremamente escasso de informações quando comparado a planos de manejo de outras UCs próximas como o do Parque Estadual da Serra da Tiririca (Aprovado pela resolução INEA No 107 de 05 de fevereiro de 2015). Essa falta de informações faz com que os gestores e a comunidade científica não tenham real dimensão do quanto se conhece dessa UC e quais as áreas que pouco ou nada se sabe. Essa informação é de extrema relevância para o planejamento de estudos futuros.

\section{CONCLUSÕES}

Algumas áreas de pesquisas tiveram trabalhos publicados todos ou quase todos os anos (fauna e flora). Outras áreas possuem uma deficiência de estudos como a Limnologia e a educação ambiental.

Para reverter este deficit em algumas áreas do conhecimehto é necessário que equipes de pesquisas que já venham realizando trabalhos semelhantes em outros locais se interessem em estudar a APA de Maricá. O presente trabalho sugere uma reformulação do plano de manejo da APA de Maricá e um maior diálogo entre os gestores da UC e os centros de pesquisa visando evitar lacunas de conhecimento sobre esta importante área de conservação.

\section{REFERÊNCIAS BIBLIOGRÁFICAS}

ANDRADE, M. A. B. A. Baixada Santista: Aspectos Geográficos. São Paulo: EDUSP, 1967.

ALENCAR, E. Inea recebe pedido para construção de resort na APA de Maricá. Blog

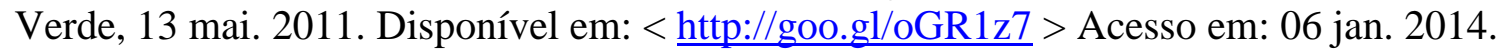

ALVAREZ, M.C.; PEREIRA, A.; ARAUJO, D. S. D.; PEREIRA, O. J. Estrutura de uma comunidade arbustiva da restinga de Barra de Maricá - RJ. Revista brasil. Bot., São Paulo, V.24, n.3, p.273-281, set. 2001.

ARAUJO, U. Projeto de criação do Parque Natural Municipal da Restinga de Maricá. Disponível em: < http://www.sineduc.com.br/sistema/arquivos/movimentos/317e1 movimen- to_pro_restinga.pdf $>$ Acesso em: 13/04/2015.

AZEVEDO M.N.S.; SERRANO C.L. Expansão Urbana e Urbanização dispersa. Duas faces da mesma moeda? Disponível em: < http://www.ppgau.uff.br/site/wp-content/uploads/ 2012/10/uff_ppgau_art_marlice.pdf > Acesso em: 13/04/2015

CAMPELLO, B. S. Encontros Científicos. In: Fontes de informação para pesquisadores e profissionais. Belo Horizonte: UFMG, 2000. 319 p.

COYUNJI, Michele Abuche. Área de proteção ambiental de Maricá e o uso sustentável: da comunidade tradicional ao megaempreendimento. Anais: Encontros Nacionais da ANPUR, v. 14, 2013. 
CRISHYNO, J.; HOLZER, W.; PIRES, A.C. Urbanização em áreas de restinga: uma proposta de avaliação de sua sustentabilidade. $X$ Encontro Nacional da Anpur: Novas escalas e estratégias territoriais na gestão ambiental. 12 p., 2003.

DA MATTA, C. Liminar favorece a proteção da restinga de Maricá. EBC Rádios, 03 dez. 2013. Disponível em: < http://goo.gl/b4XiZu > Acesso em: 06 jan. 2014.

DOMINGUES, R. Projeto de recategorização da Área de Proteção Ambiental de Maricá - RJ. Pós-Graduação em Licenciamento Ambiental, Centro Universitário de Barra Mansa, RJ. 104p. 2010.

FIOCRUZ E FASE. RJ - Pescadores artesanais da APA de Maricá lutam contra a instalação de mega resort. Disponível em: <

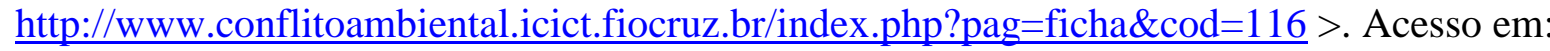
$01 / 05 / 2015$

HOLZER, W.; CRICHYNO, J.; PIRES, A. C. 2004. Sustentabilidade da urbanização em áreas de restinga: uma proposta de avaliação pós-ocupação. Paisagem Ambiente: ensaios - n. 19 - São Paulo - p. 49 - 66.

INEA, Resolução № 107 de 05 de fevereiro de 2015. Aprova o plano de manejo do Parque Estadual da Serra da Tiririca. 2015.

LOUREIRO, D. S., MATIAS, M. L.; FREIRE, D. G. Avaliação do conflito sócioambiental na APA da restinga de Maricá - RJ. XVI Encontro Nacional dos Geógrafos. Porto Alegre, pp. 10, 2010.

MATIAS, M. L.; CORREA, G. S. "R" de restinga ou de resort? O novo arranjo no setor imobiliário-turístico em Maricá, no contexto do Leste Metropolitano do Rio de janeiro. Anais XVI Encontro Nacional dos Geógrafos. Crise, práxis e autonomia: espaços de resistência e de esperanças. Espaço de Diálogos e Práticas. Porto Alegre, RS, 2010.

MATIAS, M. L.; SILVA, M. C. C.; LOUREIRO, D. S.; CORREA, G. S.; FREIRE, D. G. Disputa por território na Área de Proteção Ambiental de Maricá - Rio de Janeiro. Anais XVI Encontro Nacional dos Geógrafos. Crise, práxis e autonomia: espaços de resistência e de esperanças. Espaço de Diálogos e Práticas. Porto Alegre, RS, 2010.

MONTEIRO, F. T. Os (as) apanhadores (as) de flores e o parque nacional das semprevivas (MG): Travessias e contradições ambientais. Belo Horizonte - MG Departamento de Geografia - UFMG 236 P., 2011.

RIBAS, S. C. Parasitismo por nematóides na comunidade de lagartos na Restinga de Barra de Maricá, RJ. Dissertação de Mestrado, UERJ. Rio de Janeiro. 1995.

RIZZINI, C. M.; PEREIRA, P.; OCCHIONI, E. M. L.; AGAREZ, F. V. Considerações sobre a ocorrência de Cactaceae na Apa de Maricá, Rio de Janeiro, Brasil. Acta Botânica Brasileira. Vol.4, no.2, suppl.1, p.171-182. 1990.

ROCHA, C. F. D. Ecologia de Liolaemus lutzae (Sauria: Iguanidae) na Restinga de Barra de Maricá, RJ. Monografia de Bacharelado, Universidade do Estado do Rio de Janeiro. 104 pp. 1985.

SEMAM/IEF. Reserva da biosfera da Mata Atlântica. Rio de Janeiro: SEMAM/IEF, 1994.

UEHARA-PRADO, M.; Fonseca, R.L. Urbanization and Mismatch with Protected Areas Place the Conservation of a Threatened Species at Risk. Biotropica 39(2): 264-268. 2007. 
VRCIBRADIC, D. Diferenças ecológicas entre duas espécies simpátricas de lagartos do gênero Mabuya (Scincidae) na Restinga da Barra de Maricá - RJ. Monografia de bacharelado, Instituto de Biologia da Universidade do Estado do Rio de Janeiro, 42 p. 1992.

WINCK, G. R. Comunidades de lagartos dos remanescentes de restinga do Estado do Rio de Janeiro: Riqueza, diversidade e onde estão as espécies endêmicas e ameaçadas de extinção. Tese (Doutorado em Ecologia e Evolução) - Universidade do Estado do Rio de Janeiro, Rio de Janeiro, RJ. 2012. 